differences between patients with schizophrenia and controls generally has little effect for most brain regions - probably as a consequence of the small amount of additional data gleaned for any particular region in specific subject groups. The only region in a subject group to have more than one additional datum was the left and right temporal lobes in both genders combined. The result for this region was also changed by more than any other cortical region, from $-6 \%$ and $-9.5 \%$ (left and right) to $-3.5 \%$ and $-7 \%$. Similarly sized but opposite effects were found for the prefrontal lobes, rendering the revised median differences more compatible with those of the temporal lobes $(-5.5 \%$ and $-4 \%$, respectively). The largest overall change was for the right lateral ventricle volume in both genders, the median difference being reduced from $36 \%$ to $23 \%$ in patients with schizophrenia. The pre-specified region of maximal interest (left amygdalo-hippocampus) was not altered the only relevant data (Waldo et al, 1994) reporting these structures separately. One previous study had reported data this way, giving a new median estimate (between the two studies) of $-11.5 \%$ and $-9 \%$ (left and right) for the amygdala, and -6 and $-4 \%$ for the hippocampus.

The grey/white segmentation data in Woods \& Yurgelun-Todd (1991), that only had two relevant previous studies, were the only data that actually altered the findings. Whereas prefrontal and temporal white matter was bilaterally increased before (Lawrie \& Abukemil, 1998), such volume increases are only evident in the left temporal lobe after incorporating the new data and the other three regions are actually reduced in line with overall and grey matter reductions. However, the inclusion of one further study - in an updated review (Lawrie, 1999) - re-instates the previous finding. Overall, therefore, the main conclusions of the review - that patients with schizophrenia have small reductions in whole brain volumes as well as greater reductions in medial temporal lobe structures - remain unaltered.

What has this exercise taught us? First, systematic reviewers can fail to include relevant articles through oversight, despite doing appropriate searches. Second, full reporting of comprehensive searches is desirable - as a general rule and because unidentified articles where there are few published papers are disproportionately important. Finally, readers with good memories will remember that we staked a bottle of Glenndronnach malt whisky on the outcome of our efforts.
As there were exactly five additional articles identified (rather than more or fewer) we have declared an honourable draw.

Adams, C. E., Thornley, B. \& Joy, C. (1998) Systematic does not necessarily mean comprehensive (letter). British Journal of Psychiatry, 172, 450-45I.

American Psychiatric Association (1987) Diagnostic and Statistical Manual of Mental Disorders (3rd edn, revised) (DSM-III-R). Washington, DC: APA.

Cowell, P. E., Kostianovsky, D. J., Gur, R. C., et al (1996) Sex differences in neuroanatomical and clinical correlations in schizophrenia. American Journal of Psychiatry, 153, 799-805.

DeLisi, L. E., Hoff, A. L., Kushner, M., et al (1992) Left ventricular enlargement associated with diagnostic outcome of schizophreniform disorder. Biological Psychiatry, 32, 199-201.

Lawrie, S. M. (1998) Comprehensiveness of systematic review (letter). British Journal of Psychiatry, 173, 87-88.

- (1999) Neuropathology and brain imaging in schizophrenia. In Schizophrenia: Concepts and Clinical Management (eds E. C. Johnstone, M. Humphries, F. Lang et al), pp. 70-128. New York: Cambridge University Press.

_ \& Abukmeil, S. (1998) Brain abnormality in schizophrenia. A systematic and quantitative review of volumetric magnetic resonance imaging studies. British journal of Psychiatry, 172, I10-120.

Lim, K. O., Beal, M., Harvey, R. L., et al (1995) Brain dysmorphology in adults with congenital rubella plus schizophrenia-like symptoms. Biological Psychiatry, 37, 764-776.

Waldo, M. C., Cawthra, E., Adler, L. E., et al (1994) Auditory sensory gating, hippocampal volume, and catecholamine metabolism in schizophrenics and their siblings. Biological Psychiatry, 12, 93-106.

Woods, B.T. \& Yurgelun-Todd, D. (1991) Brain volum loss in schizophrenia: when does it occur. Schizophrenia Research, 5, 202-204.

S. M. Lawrie University Department of Psychiatry, Royal Edinburgh Hospital, Edinburgh EHI0 5HF

C. E. Adams, B. Thornley, C. Joy Cochrane Schizophrenia Group, Middle Way, Summertown, Oxford OX2 7LG

\section{PACT to the future}

The PRiSM papers (Becker et al, 1998) and subsequent editorial (Marshall et al, 1999) on assertive community treatment (ACT) highlight the quest by mental health professionals to provide appropriate and effective services to vulnerable individuals with severe mental illness. The design, implementation and model fidelity of ACT have been a widely researched aspect of community mental health care (Mueser et al, 1998).

Interestingly, the studies and editorials animatedly examine research carried out between 1992 and 1994, the inception and teething stage of the Nunhead psychiatric assertive community care team (PACT). Crucially, this period was characterised by staff and client recruitment, site relocation, and resource allocation while imbibing the tenets of ACT. Designers of ACT fidelity measures (McGrew et al, 1994) sound a note of caution that "implementation and fidelity are developmental" and this "natural temporal evolution in service" if not accounted for in research, can be a potential source of unexplained error.

The Nunhead PACT team has shown considerable development over the 1990s derived from tailoring a service to suit its own unique client population. Community mental health services are not identical as they cover unique geographical and socio-economic areas, with diverse ethnic, demographic and psychopathological characteristics. However, numerous studies of ACT facilities (mainly outside the UK) stress that model fidelity is fundamental to effective ACT service provision (Teague $e t$ al, 1998).

We present here an updated description of the Nunhead PACT team. The multicultural client population $(55 \%$ male, $45 \%$ female), with a mean age of 46 years, has a predominant diagnosis of schizophrenia $(80 \%)$ and the remainder affective psychoses. With a mean duration of illness of 17 years, characterised by multiple hospitalisations, admissions are currently onesixth pre-ACT intervention levels.

The team comprises staff with diverse backgrounds namely psychiatrists, clinical psychologist, psychiatric nurses, social workers, a community forensic psychiatric nurse, an occupational therapist and support workers, with a staff (keyworker) : client ratio of $1: 12$. Furthermore, regular input from a benefits/welfare adviser, chaplain and community pharmacist has proven beneficial to clients and their carers. Dual diagnosis/substance misuse expertise is also being developed. The mean core staff duration in the team is 4.5 years. Patient input through client-led weekly community meetings and newsletters has been found to be invaluable. A variety of work rehabilitation activities (e.g. computer skills, tool workshop, photography and college courses) are being taken up by clients. Multi-sectoral collaboration with voluntary and statutory housing befriending, and ethnic and religious agencies has also facilitated engaging clients in the community. With its current resource and near total programme fidelity, is it likely that the service is actually more 
advanced than many other ACT services? What intervals and outcome measures reliably assess ACT interventions? In our opinion, two years is a relatively short period to adequately engage, treat and initiate significant rehabilitation of a person with severe mental illness. We owe it to our clients to enhance their quality of life by sustaining the merits of PACT into the 21st century. Prospective longitudinal research may still identify elements crucial to advancing lessons of the past to the future.

\section{Becker, T., Holloway, F., McCrone, P., et al (1998)} Evolving service interventions in Nunhead and Norwood. PRiSM Psychosis Study 2. British Journal of Psychiatry, I73, 37I-375.

Marshall, M., Bond, G., Stein, L. L., et al (1999) PRiSM Psychosis Study. Design limitations, questionable conclusions. British Journal of Psychiatry, I75, 50I-503.

McGrew, J. H., Bond, G. R., Dietzen, L. L., et a (1994) Measuring the fidelity of implementation of a mental health program model. Journal of Consulting and Clinical Psychology, 62, 670-678.

Mueser, K. T., Bond, G. R., Drake, R. E., et al (1998) Models of community care for severe mental illness: a review of research on case management. Schizophrenic Bulletin, 24, 37-74.

Teague, G. R., Bond, G. R. \& Drake, R. E. (1998) Program fidelity in assertive community treatment: development and use of a measure. American Journal of Orthopsychiatry, 68, 216-232.

O. A. Ayonrinde, J. Sauer

F. Macdiarmid PACT Team, Maudsley Hospital, Denmark Hill, London SE5 8AZ

\section{Drug treatment for resistant depression}

On the basis of a four-week study carried out in 122 patients suffering from treatment-resistant depression, Poirier \& Boyer (1999) claimed that "venlafaxine showed some evidence of superiority to paroxetine in this difficult-to-treat population". Careful analysis of their results, however, suggests that evidence supporting this assertion can be improved.

First, it should be noted that the design of the study was inherently biased in favour of venlafaxine since, in both treatment groups, two-thirds of included patients had proved to be "resistant" to a selective serotonin reuptake inhibitor (SSRI). In spite of this, no significant differences were observed between venlafaxine and paroxetine for the primary efficacy variable (defined as the change in total Hamilton Depression Rating Scale (HAM-D) score between day 0 and day 28) in either the observed case analysis $(-11.1$ and -10.2 respectively; $P=0.55$ ) or the last-observation-carried-forward (LOCF) analysis ( $P=0.70$, intention-to-treat).

Furthermore, there was no significant difference between the two treatments with respect to the response rates $(>50 \%$ decrease from baseline in HAM-D score and a Clinical Global Impression (CGI) improvement score of 1 or 2) following the more robust LOCF analysis, although for the observed case analysis the difference just achieved significance $(P=0.044)$.

Second, CGI severity and improvement scores improved over time following both treatments. Although there was no significant difference between the two groups, the trend was clearly in favour of paroxetine.

Finally, it should be noted that the dose titration for paroxetine was very rapid (30 mg as early as on Day 5) and neither optimal nor consistent with the manufacturer's recommendations. This rapid titration could have contributed to the high incidence of adverse events found in the paroxetine-treated group $(63 \%$ of patients treated with paroxetine compared with $69 \%$ of those given venlafaxine). In addition, it appears that the comparison was not performed at equivalent doses for both antidepressants; the mean daily dose of venlafaxine was $269 \mathrm{mg} /$ day (i.e. $44 \mathrm{mg} /$ day more than the maximal daily dose recommended by the manufacturer in ambulatory patients) $v .36 .3 \mathrm{mg}$ for paroxetine, which is not the maximal dose for this agent.

To sum up, the authors emphasis on a fairly marginal significance emerging from a subsidiary analysis of a secondary efficacy parameter seems disproportionate.

Poirier, M.-F. \& Boyer, P. (1999) Venlafaxine and paroxetine in treatment-resistant depression. Doubleblind, randomised comparison. British Journal of Psychiatry, 175, 12-16

S. Daniels SmithKline Beecham Pharmaceuticals, Neurosciences Therapeutic Unit, New Frontiers Science Park (South), Third Avenue, Harlow, Essex CMI9 5AW

Author's reply: We do not consider that our study was "inherently biased in favour of venlafaxine" for three main reasons:

(a) The proportion of two-thirds of patients included in each group who were previously resistant to treatment with an SSRI is a realistic picture of what is observed in everyday practice, since the prescription of an SSRI is now the predominant one in any type of depression. (b) Two-thirds of the patients included in the venlafaxine (a serotonin and noradrenaline reuptake inhibitor) group were previously resistant to tricyclic antidepressant drugs, which also act on noradrenaline and serotonin. The bias in favour of venlafaxine is in the same proportion as the bias in favour of paroxetine.

(c) There is no clear evidence that a patient resistant to an SSRI must not be switched to another SSRI. Not all SSRIs are the same, and the consistent pharmacological differences between these drugs authorise our point of view for such a switch.

What is more, Dr Daniels' opinion, that when a patient is resistant to an SSRI subsequent treatment with paroxetine (another SSRI) should be avoided, is likely to be incorrect as in our study, a significant number of patients previously resistant to an SSRI afterwards responded to treatment with paroxetine.

The fact that no significant differences were observed between venlafaxine and paroxetine with respect to the mean HAM-D change, both in the observed-case and in the LOCF analyses, was fully recognised in our report. The main differences we reported between the two drugs was in remission rate - an important criterion for prediction of future outcome.

Finally, regarding the dosages of the drugs used, at the time the study protocol was designed, paroxetine dosage (including dose titration) was not very clear in terms of regulatory recommendations (in France at least) and it was not possible to recommend a dosage of paroxetine as high as $40 \mathrm{mg} /$ day. This can be seen as too low now, in the light of subsequent research on the dose-response relationship for paroxetine.

M.-F. Poirier Centre Hospitalier Sainte-Anne, I, rue Cabanis, 75674 Paris Cedex 14, France

\section{Cholesterol, depression and suicide}

In a recent study low serum total cholesterol was associated with an increased risk of suicide (Partonen et al, 1999). However, the study population was a special subgroup, since the subjects were older male smokers. In addition, the final trial participants were very selected, since the target population included approximately 283000 subjects, but 